\title{
Métodos numéricos aplicados a cubagem de madeira
}

\author{
Gustavo José Neves da Silva ${ }^{1}$ \\ Mauricio Aronne Pillon ${ }^{1}$ \\ Volnei Avilson Soethe ${ }^{2}$ \\ ${ }^{1}$ Departamento de Ciência da Computação \\ Universidade do Estado de Santa Catarina (UDESC) - Joinville, SC - Brasil \\ ${ }^{2}$ Departamento de Matemática \\ Universidade do Estado de Santa Catarina (UDESC) - Joinville, SC - Brasil \\ gustavo.neves@yandex.com, mauricio.pillon@udesc.br, volneidalethos.com.br
}

\begin{abstract}
The purpose of this work is to design a tool for calculating wood cubes, based on numerical methods, using parallel approaches to improve the accuracy of the results. For this purpose, the task parallelism strategy will be applied, in which each work unit will be responsible for calculations related to a task of the numerical integration algorithm.
\end{abstract}

Resumo. A proposta deste trabalho é a concepção de uma ferramenta para o cálculo da cubagem de madeira, baseada em métodos numéricos, utilizando abordagens paralelas visando aprimorar a precisão dos resultados. Para tanto será aplicada a estratégia de paralelismo de tarefas, na qual cada unidade de trabalho será responsável pelos cálculos relativos a uma tarefa do algoritmo de integração numérica.

\section{Introdução}

De acordo com o levantamento Produção da Extração Vegetal e da Silvicultura (Pevs) [Instituto Brasileiro de Geografia e Estatística 2018a], o Brasil possui 9,89 milhões de hectares de florestas plantadas. No estado Paraná e no de Santa Catarina a espécie predominante utilizada na silvicultura é o Pinus Taeda, com respectivamente $801.316(53,38 \%$ da área cultivada no estado) e 609.872(63,11\% da área cultivada no estado) hectares cultivados. No que se refere, exclusivamente, ao cálculo do volume de produção por lote, que é subdividido em períodos, a forma de automatizar o processo passa pela modelagem matemática do cálculo geométrico das toras. O inventário florestal, para fins comerciais, é viabilizado pelo cálculo da estimativa do volume das toras, as quais possuirão diversos destinos como apresentado na Tabela 1. Métodos analíticos

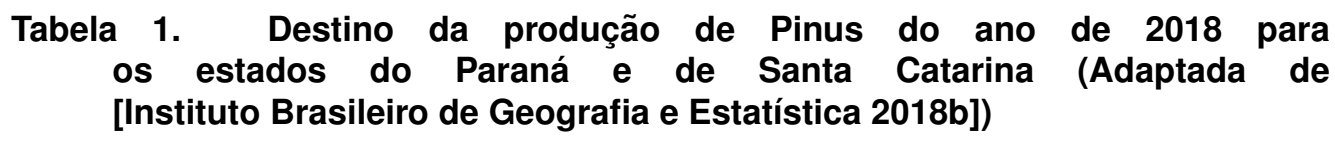

\begin{tabular}{llll}
\hline \multirow{2}{*}{ Lenha $\left(m^{3}\right)$} & \multicolumn{2}{c}{ Madeira em tora $\left(m^{3}\right)$} \\
\cline { 3 - 4 } & & Papel e Celulose & Outras finalidades \\
\hline Paraná & 1.385 .245 & 8.430 .773 & 11.349 .215 \\
\hline Santa Catarina & 1.707 .603 & 6.1061 .93 & 8.274 .914 \\
\hline
\end{tabular}


Tabela 2. Estimação do volume de toras (Adaptada de [Briggs 1994])

\begin{tabular}{ll}
\hline \multicolumn{1}{c}{ Método } & \multicolumn{1}{c}{ Fórmula(V) } \\
\hline Smalian [Briggs 1994, Governo de Alberta 2017, Smith 2016, Benoni Kewilaa 2017] & $V=f\left(d_{s}^{2}+d_{l}^{2}\right) L / 2$ \\
Bruce's butt log [Briggs 1994, Smith 2016, Benoni Kewilaa 2017] & $V=f\left(0.75 d_{s}^{2}+0.25 d_{l}^{2}\right) L / 2$ \\
Huber [Briggs 1994, Smith 2016] & $V=f d_{m}^{2} L$ \\
Sorenson [Briggs 1994, Mata Nativa 2019] & $V=f\left(d_{s}+0.05 L\right)^{2} L$ \\
Newton [Briggs 1994, Smith 2016] & $V=f\left(d_{s}^{2}+4 d_{m}^{2}+d_{l}^{2}\right) L / 6$ \\
Subneiloid [Briggs 1994, Smith 2016, Benoni Kewilaa 2017] & $V=f\left[\left(d_{s}+d_{l}\right) / 2\right]^{2} L$ \\
Two-end conic [Briggs 1994, Hart 2013, Smith 2016] & $V=f\left(d_{s}^{2}+d_{s} d_{l}+d_{l}^{2}\right) L / 3$ \\
\hline
\end{tabular}

são comumente empregados pelas indústrias do setor para o cálculo dessa estimativa. Esses métodos permitem estimar o volume de uma tora por meio do cálculo do volume de um cilindro, cone ou paraboloide. Portanto, a acurácia da estimativa do volume depende diretamente da proximidade geométrica da forma real da tora em relação a forma modelada pela técnica. O cálculo do volume dos métodos analíticos diferem de acordo com as fórmulas apresentadas na Tabela 2, a qual considera: $f=0,005454$ (sistema imperial) ou 0,00007854 (sistema métrico); $V=$ volume, em pés cúbicos ou em metros cúbicos; $d_{s}, d_{m}, d_{l}=$ menor, médio e maior diâmetros, em polegadas ou em centímetros; e $L=$ comprimento, em pés ou em metros.

Todavia, assumir que toras reais sejam formas geométricas perfeitas, como supõem as técnicas de estimativa com métodos analíticos, é uma escolha arriscada pois os cálculos realizados podem produzir erros de diferentes magnitudes, uma vez que há dificuldade em representar com fidelidade a forma real da tora. $\mathrm{O}$ uso da integração numérica, dada sua natureza adaptativa, aprimora a precisão dos resultados oriundos do cálculo do volume, se comparado com métodos analíticos. A integração numérica caracteriza-se pela formação de uma função contínua $f(x)$ em um intervalo $[a, b]$, então, existe $F(x)$, tal que: $F^{\prime}(x)=f(x)$. Substituindo-se a função $f(x)$ por um polinômio que a aproxime dos valores do intervalo $[a, b]$, o que caracteriza um método numérico, viabiliza a aplicação da função contínua ao cálculo de volume de toras. Uma das possibilidades para o cálculo da integral é a abordagem proposta pelo método dos trapézios e suas derivações. O método dos trapézios tem o erro determinado por $-\frac{h^{3}}{12} f^{\prime \prime}(\xi)$, e é descrito pela aproximação da $\int_{a}^{b} f(x)$, tal que $x_{0}=a, x_{1}=b, h=b-a$, resultando em: $\int_{a}^{b} f(x) d x=\frac{h}{2}\left[f\left(x_{0}\right)+f\left(x_{1}\right)\right]-\frac{h^{3}}{12} f^{\prime \prime}(\xi)$. No contexto do trabalho, o volume da tora pode ser aproximado pela seguinte integral: $\int_{h_{0}}^{h_{m}} \int_{r_{0}}^{r_{m}} \pi r^{2} h d r d h$, na qual, $h_{0}$ e $h_{m}$, representam o intervalo de integração referente a altura e, $r_{0}$ e $r_{m}$, o intervalo de integração referente ao raio. Porém, com intervalos de integração extensos, no contexto do trabalho representam as deformidades da tora, o método dos trapézios apresenta imprecisão nos resultados do cálculo. A solução utilizada foi o método dos trapézios compostos, o qual subdivide o intervalo de integração e aplica o método dos trapézios repetidas vezes. Da seguinte forma, tendo os pontos de subdivisão do intervalo $[a, b]$ como $x_{i}$, tais que $x_{i+1}-x_{i}=h$ e $i=0,1, \ldots, m-1$, obtém se: $\int_{x_{0}}^{x_{m}} f(x) d x=\frac{h}{2}\left[f\left(x_{0}\right)+2 f\left(x_{1}\right)+2 f\left(x_{2}\right)+\cdots+2 f\left(x_{m-1}\right)+f\left(x_{m}\right)\right]-m h^{3} \frac{f^{\prime \prime}(\xi)}{12}$ Por fim, ocorre a aplicação da extrapolação de Richardson ao método dos trapézios compostos resultando no método de Romberg. A extrapolação pode ser aplicada sempre que é conhecido que a técnica de aproximação tem um termo de erro com uma forma previsível(aquela que depende de um parâmetro), ou seja, $E(h)=C h^{p}$, tal que $C$ é uma constante e $p$ é o expoente do termo do erro e $h$ é o parâmetro. Logo a aproximação( $G)$ 
para uma $g(h)$ é dada por $G=g(h)+E(h)$. Para dois parâmetros $h_{1}$ e $h_{2}$ a aproximação é definida por: $G=\frac{2^{p} g\left(\frac{h_{1}}{2}\right)-g\left(h_{1}\right)}{2^{p}-1}$. Na abordagem proposta pelo método de Romberg apenas a primeira coluna da matriz de solução necessita do cálculo por meio do método dos trapézios compostos, os outros membros da matriz são calculados por meio aplicação da extrapolação de Richardson, ou seja, uma média ponderada dos outros componentes.

\section{Solução Proposta}

O presente trabalho tem como proposta a paralelização do método de Romberg e aplicar o mesmo ao cálculo do volume de madeira. A paralelização tem como base a técnica de paralelismo de tarefas e foi aplicada a arquiteturas paralelas com memória compartilhada, de forma que as funcões internas do método de integração foram particionados em múltiplas threads. A linguagem $\mathrm{C}$ foi utilizada nas implementações sequenciais dos métodos analíticos e na paralela para o método de Romberg, a qual foi utilizada para a criação de threads a biblioteca POSIX Threads. A plataforma de testes foi uma máquina SMP (processador AMD A4-5000 com 4 núcleos e 4GB de memória), sistema operacional ARCH Linux e compilador GCC 9.2.0. Como resultado foi obtida a Figura 1, na qual é demostrado o volume relativo do erro resultante da aplicação dos métodos à um lote de cultivo (1667 toras) com volume real de $0.28369 \mathrm{~m}^{3}$, no qual cada tora foi representada por um tronco de cone.

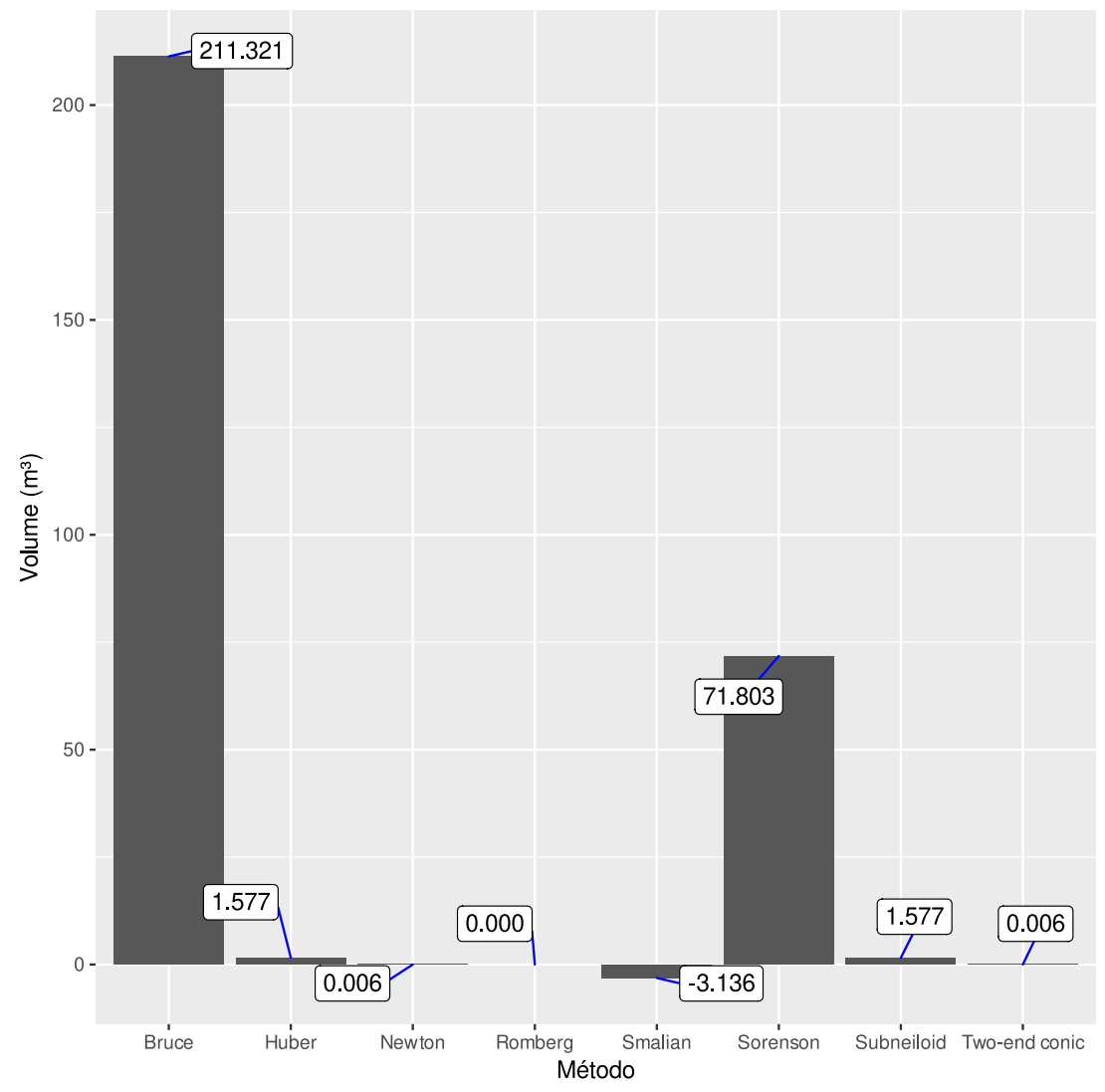

Figura 1. Volume resultante do erro para 1667 árvores. Fonte: Elaborada pelo autor 
Tabela 3. Resultados dos métodos analíticos e do método de Romberg

\begin{tabular}{llllllll}
\hline & Min.(s) & $1^{\circ}$ Qu.(s) & Mediana(s) & Média(s) & $3^{\circ}$ Qu.(s) & Max.(s) & Volume $\left(m^{3}\right)$ \\
\hline Smalian & 0.0001400 & 0.0001485 & 0.0001520 & 0.0001533 & 0.0001560 & 0.0001980 & 0.28557144 \\
Bruce & 0.00000300 & 0.00000375 & 0.00000400 & 0.00000380 & 0.00000400 & 0.00000500 & 0.15692292 \\
Huber & 0.0000030 & 0.0000030 & 0.0000030 & 0.0000033 & 0.0000040 & 0.0000040 & 0.28274400 \\
Sorenson & 0.00000300 & 0.00000300 & 0.00000300 & 0.00000325 & 0.00000325 & 0.00000400 & 0.24061691 \\
Newton & 0.0000030 & 0.0000030 & 0.0000030 & 0.0000033 & 0.0000040 & 0.0000040 & 0.28368648 \\
Subneiloid & 0.00000300 & 0.00000300 & 0.00000300 & 0.00000375 & 0.00000400 & 0.00001300 & 0.28274400 \\
Two-end conic & 0.0000030 & 0.0000030 & 0.0000030 & 0.0000032 & 0.0000030 & 0.0000040 & 0.28368648 \\
Romberg Sequencial & 0.004009 & 0.004804 & 0.005427 & 0.005571 & 0.006305 & 0.007518 & 0.28369 \\
Romberg phtreads & 0.004936 & 0.004982 & 0.005075 & 0.005140 & 0.005238 & 0.005736 & 0.28369 \\
\hline
\end{tabular}

\section{Considerações Finais}

Erros resultantes do cálculo de volume de toras causam estimativas incorretas e portanto prejuízos ao setor de silvicultura. Como demostrado nesse trabalho, a versão paralelizada por meio do uso da biblioteca POSIX Threads possibilitou a validação da proposta do particionamento do problema. Porém tal proposta demonstrou não ser escalável, uma vez que, apesar de ter ocorrido a minimização do erro no cálculo do volume de toras, em comparação ao apresentado pelos métodos analíticos, também ocorreu um aumento no tempo de processamento em comparação a esses, como apresentado na Tabela 3. Como trabalhos futuros, o algoritmo paralelo será portado para uma arquitetura vetorial (SIMD Single Instruction, Multiple Data) e são pretendidos outros experimentos com toras reais.

\section{Referências}

Benoni Kewilaa, S. M. K. (2017). The effect of different methods to determine log volume and their impacts to veneer recovery. Modern Environmental Science and Engineering, 3(5):302-308.

Briggs, D. (1994). Forest products measurements and conversion factors: With special emphasis on the us pacific northwest college of forest resources. University of Washington Seattle, WA.

Governo de Alberta (2017). Alberta Scaling Manual - Scaling methods: Smalian Scale. Governo de Alberta, Alberta, Canadá. Acessado em: Jul. 2019.

Hart, N. (2013). Converting between Western North American log scaling methods. Jendro And Hart, LLC, Sunriver, Oregon. Acessado em: Ago. 2019.

Instituto Brasileiro de Geografia e Estatística (2018a). Levantamento produção da extração vegetal e da silvicultura. Acessado em: Oct. 2019.

Instituto Brasileiro de Geografia e Estatística (2018b). Tabela 291 - quantidade produzida e valor da produção na silvicultura, por tipo de produto da silvicultura. Acessado em: Oct. 2019.

Mata Nativa (2019). Acessado em: Ago. 2019.

Smith, D. A. M. (2016). Forest measurements and inventory (for 274). notas de aula. Acessado em: Ago. 2019. 